\title{
基于篮分水平浓度法的洞庭湖沉积物质量基准初步分析”
}

\author{
赵艳民 ${ }^{1}$, 张 雷 $^{1}$, 王丽婧 ${ }^{1}$, 秦延文 ${ }^{* * *}$, 郑丙辉 ${ }^{1}$, 马迎群 ${ }^{1}$, 迟明慧 ${ }^{1}$, 刘志超 ${ }^{1}$, 杨晨晨 ${ }^{1}$, 李利强 ${ }^{2}$ \\ (1: 中国环境科学研究院环境基准与风险评估国家重点实验室,北京 100012) \\ $(2:$ 洞庭湖生态环境监测中心, 岳阳 414000)
}

\begin{abstract}
摘 要: 基于 2013-2015 年调查获取的 129 个洞庭湖表层沉积物和底栖生物的调查数据,应用篎分水平浓度法初步探 讨了洞庭湖沉积物几种重金属沉积物质量基准的推荐值. 结果表明,洞庭湖沉积物砷 $(\mathrm{As}) 、$ 镉 $(\mathrm{Cd}) 、$ 铬 $(\mathrm{Cr}) 、$ 铜 $(\mathrm{Cu}) 、$ 铅 $(\mathrm{Pb})$ 和锌 $(\mathrm{Zn})$ 含量均值为 $17.2 、 2.63 、 56.9 、 32.4 、 38.4$ 和 $96.3 \mathrm{mg} / \mathrm{kg}(\mathrm{DW})$; 篮分水平浓度法获取的洞庭湖沉积物 $\mathrm{As} 、 \mathrm{Cd}$ 、 $\mathrm{Cr} 、 \mathrm{Cu} 、 \mathrm{~Pb}$ 和 $\mathrm{Zn}$ 质量基准分别为 11.97、1.13、42.03、19.17、23.63 和 $67.54 \mathrm{mg} / \mathrm{kg}(\mathrm{DW})$. 通过与不同国家及地区指定的重 金属质量基准推荐值以及本地区采用其他方法推导的重金属基准推荐值进行比较,推算出本研究获取的 6 种重金属沉积 物质量基准推荐值接近所有数据的中间值. 对于洞庭湖流域,不同方法获取的沉积物重金属质量基准具有可比性. 然而, 鉴于用于推导沉积物质量基准的数据采集涉及的区域较为有限, 且除了重金属污染外, 影响底栖生物分布的因素较为复 杂, 导致获取的沉积物重金属质量基准推荐值存在一定的不确定性.
\end{abstract}

关键词: 洞庭湖; 重金属;沉积物质量基准;篮分水平浓度法

\section{Sediment quality criteria for heavy metals in Lake Dongting based on screening level con- centration approach}

ZHAO Yanmin $^{1}$, ZHANG Lei ${ }^{1}$, WANG Lijing ${ }^{1}$, QIN Yanwen ${ }^{1 * *}$, ZHENG Binghui ${ }^{1}$, MA Yingqun ${ }^{1}$, CHI Minghui $^{1}$, LIU Zhichao ${ }^{1}$, YANG Chenchen ${ }^{1} \&$ LI Liqiang ${ }^{2}$

(1: State Key Laboratory of Environmental Criteria and Risk Assessment, Chinese Research Academy of Environmental Sciences, Beijing 100012, P.R.China)

(2: Dongting Lake Eco-Environment Monitoring Centre, Yueyang 414000, P.R.China)

Abstract: 129 surface sediment samples and the co-occurrence macrozoobenthos samples were collected from Lake Dongting during 2013-2015. Concentrations of six kinds of heavy metal-arsenic (As), cadmium (Cd), chromium ( Cr), copper ( Cu), lead $(\mathrm{Pb})$ and zinc $(\mathrm{Zn})$ in the sediment were measured and the species composition of macrozoobenthos was identified. The results showed that the average concentrations of $\mathrm{As}, \mathrm{Cd}, \mathrm{Cr}, \mathrm{Cu}, \mathrm{Pb}$ and $\mathrm{Zn}$ were 17.2, 2.63, 56.9, 32.4, $38.4 \mathrm{and} 96.3 \mathrm{mg} / \mathrm{kg}$ (DW), respectively. Based on the field data on the co-occurrence of sediments and macrozoobenthos and the concentrations of heavy metal, the screening level concentration (SLC) approach was induced to preliminarily explore the sediment quality criteria (SQC) recommended values of heavy metal for surface sediments from Lake Dongting. The calculated SQCs of As, Cd, Cr, Cu, $\mathrm{Pb}$ and $\mathrm{Zn}$ of Lake Dongting were 11.97, 1.13, 42.03, 19.17, 23.63 and $67.54 \mathrm{mg} / \mathrm{kg}$ (DW), respectively. These SQC values were compared with those of different countries and regions. It was found that SQCs obtained in this study were close to the median values of the reference data. The SQCs of heavy metal in Lake Dongting came from different derivation methods were comparative. However, there is still some uncertainty when applied the values of SQC derived from screen level concentration approach. One reason responsible for the uncertainty of the SQCs is that the field data used to derive SQCs is limited. In addition of heavy metal pollution, many other factors can affect the distribution of macrozoobenthos, which will lead to relatively biased in the values of SQCs. Keywords: Lake Dongting; heavy metal; sediment quality criteria; screening level concentration approach

* 国家重点基础研究发展计划项目 (2012CB417004) 和国务院三峡办洞庭湖江湖生态监测项目 (JJ [ 2015]-002) 联合 资助. 2016-01-29 收稿; 2016-04-13 收修改稿. 赵艳民(1979 ), 男, 博士, 副研究员; E-mail: zhaoym@ craes. org.cn.

** 通信作者;E-mail: Qinyw@ craes.org.cn. 
湖泊沉积物是流域内各种污染物质的蓄积库,重金属等污染物经过水体颗粒物的吸附、络合、螯合等方 式与悬浮颗粒物结合并最终沉降于沉积物中, 因此湖泊沉积物记录了湖区环境变化的丰富信息, 在一定程 度上反映了流域人类开发活动对湖泊环境的影响 ${ }^{[1-2]}$. 由于重金属污染具有难降解、生物累积和食物链放大 等生态环境效应,成为湖泊水环境中备受关注的污染物 ${ }^{[3]}$.

沉积物质量基准 ( sediment quality criteria, SQC) 是特定的化学物质在沉积物中不对底栖生物产生不利 影响的保护性临界水平 ${ }^{[4]}$, 是底栖生物剂量-效应关系的反映 ${ }^{[5]}$, 也是进行沉积物质量评价的依据. 自美国 于 1980s 开展沉积物质量基准研究工作以来,北美、欧洲以及澳大利亚、新西兰和中国香港等国家和地区先 后采用不同的方法构建了淡水沉积物质量基准,其中一些已经作为临时性标准被环境管理部门采用 ${ }^{[6]}$. 然 而, 现有沉积物质量基准应用目的、保护目标、保护程度和基准的建立方法不同, 加之沉积物污染物毒性受 到诸如自身存在形态、沉积物粒度、有机碳等众多复杂因素的影响,使得各种沉积物环境质量基准之间存在 较大差异 ${ }^{[7]}$,对于同种重金属, 不同国家和组织之间的基准值之间差异超过几十倍 ${ }^{[8]}$, 因此难以直接套用国 外沉积物质量基准应用于我国沉积物质量评价.

1990 s 我国学者开始从不同角度介绍了国际对于沉积物质量基准的研究进展 ${ }^{[9-11]}$. 近年来, 不同研究者 开始采用不同方法开展沉积物中重金属 ${ }^{[12-13]}$ 、有机污染物 ${ }^{[14-15]}$ 基准推导方法研究. 目前沉积物质量基准的 推导方法超过 10 余种, 其中影响较大的包括背景值法 (sediment background approach, SBA)、相平衡分配法 (equilibrium partitioning approach, EqP)、沉积物加标毒性实验法 (spiked sediment bioassay approach, SSBA)、 篮分水平浓度法 ( screening level concentration approach, SLCA)、沉积物质量效应三元法 ( sediment quality triad approach, SQTA) 等 ${ }^{[16]}$.

篮分水平浓度法是基于污染物的生物效应推导保护底栖生物免受污染物危害的沉积物质量基准方法, 最初由 Neff 等 ${ }^{[17]}$ 提出用于建立美国海洋以及淡水沉积物非极性有机污染物的环境质量基准. 赵艳民等 ${ }^{[18]}$ 对篮分水平浓度法进行了详细介绍, 并应用篮分水平浓度法获取了浑河沉积物铜、铅、锌、镉 4 种重金属的 基准值.

洞庭湖 $\left(28^{\circ} 44^{\prime} \sim 29^{\circ} 35^{\prime} \mathrm{N}, 111^{\circ} 53^{\prime} \sim 113^{\circ} 05^{\prime} \mathrm{E}\right)^{[19]}$ 位于湖南省东北部, 长江中游荆江段南岸, 北通过松滋 口、太平口、藕池口 (三口) 承接长江来水, 西南部则有湘、资、沅、澧水 (四水) 来水, 湖水经由东北部的城陵 矶汇人长江, 是我国五大淡水湖之一. 湖南省是我国著名的“有色金属之乡”, 主要人湖河流之一的湘江流域 更是我国有色金属开采和冶炼重要基地,沿江两岸工矿企业排放到湘江的废水中富含镉 $(\mathrm{Cd})$ 、铜 $(\mathrm{Cu}) 、$ 、铅 $(\mathrm{Pb})$ 和锌 $(\mathrm{Zn})$ 等重金属元素 ${ }^{[20]}$, 最终汇人洞庭湖, 导致洞庭湖水系重金属健康风险不断增加 ${ }^{[21]}$. 近年来, 涉及洞庭湖沉积物重金属的研究日益增多 ${ }^{[19,22-23]}$, 但对于洞庭湖沉积物中重金属的基准研究尚未见报道.

本研究利用 2013-2015 年获取的洞庭湖沉积物和底栖生物调查数据, 应用篮分水平浓度法推导洞庭湖 沉积物中砷 $(\mathrm{As}) 、 \mathrm{Cd} 、$ 铬 $(\mathrm{Cr}) 、 \mathrm{Cu} 、 \mathrm{~Pb}$ 和 $\mathrm{Zn}$ 的质量基准值, 为制定沉积物质量标准、开展水环境综合管理提 供技术支持.

\section{1 材料与方法}

\section{1 样品采集及保存}

$2013-2015$ 年采用 $1 / 16 \mathrm{~m}^{2}$ 的改良彼得逊采泥器共收集 129 个洞庭湖表层沉积物以及底栖生物样品, 采样涉及的区域如图 1, 每个湖区单次采集 3 9 个样本, 采样深度约为 $0 \sim 10 \mathrm{~cm}$. 沉积物样品装人密封袋中 置于 $4^{\circ} \mathrm{C}$ 低温保存, 用以进行理化分析. 大型底栖动物利用分样篮 (60 目) 篮选, 标本采用 $75 \%$ (体积分数) 乙醇保存.

\section{2 样品分析与质量控制}

沉积物在通风的室内自然风干、除杂、混合均匀后, 用木棒和玛瑙研针磨碎, 过 100 目尼龙篮后采用 $\mathrm{HNO}_{3}-\mathrm{HF}\left(\mathrm{V}: \mathrm{V}=5: 1\right.$ ) 微波消解, 电热板赶酸, $2 \%$ (质量分数) $\mathrm{HNO}_{3}$ 定容至 $100 \mathrm{ml}$, ICP-MS(Agilent 7500CX) 测定. 大型底栖动物样品在解剖镜和显微镜下进行鉴定和数量统计, 获取的大型底栖动物大部分鉴定到种, 少数鉴定到属, 详细记录各物种的数目, 最后换算成每平方米的个体数目.

分析实验过程中每批样品均做全程空白, 以消除样品处理以及测定过程中可能带人的污染. 同步分析 


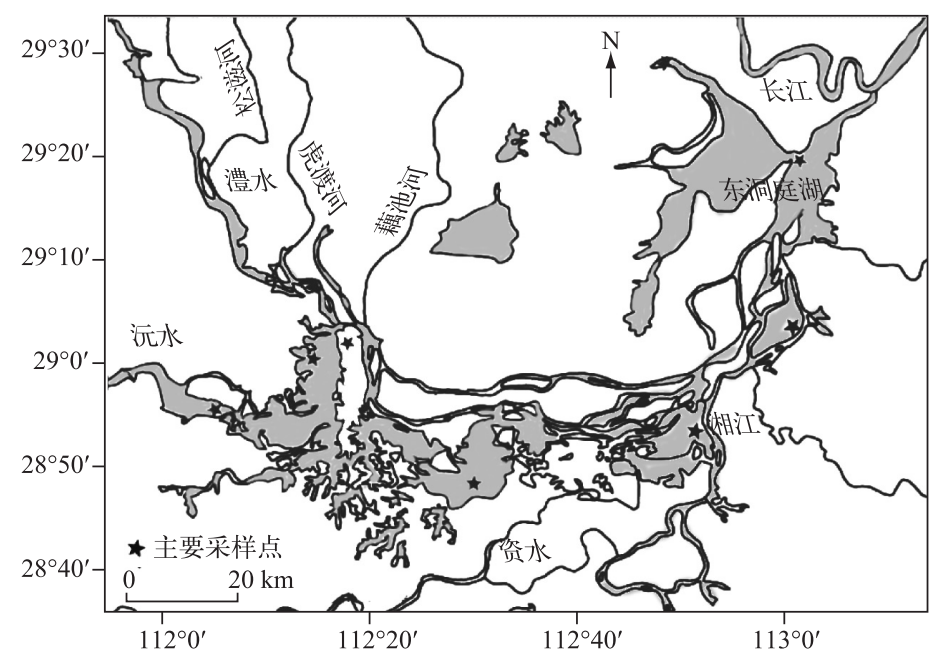

图 1 洞庭湖典型采样区域

Fig. 1 Sampling area in Lake Dongting

了购自中国地质科学院地球物理地球化学勘查研究所生产的水系沉积物成分分析标准物质 (GBW07309), 元素 $\mathrm{As} 、 \mathrm{Cd} 、 \mathrm{Cu} 、 \mathrm{Cr} 、 \mathrm{~Pb}$ 和 $\mathrm{Zn}$ 的回收率分别为 $93 \% 、 87 \% 、 84 \% 、 98 \% 、 89 \%$ 和 108\% , 均在美国国家环境保护局 (USEPA) 要求范围 $(80 \% \sim 120 \%$ ) 之内, 所有样品均进行平行样测定, 相对标准偏差均小于 $5 \%$.

\section{3 数据分析}

使用 Excel 软件对数据进行整理. 使用 Origin 8.0 软件进行正态性检验等相关统计分析, 同时进行相关 绘图.

\section{2 结果}

\section{1 洞庭湖沉积物重金属含量水平}

本研究中洞庭湖表层沉积物 $\mathrm{As} 、 \mathrm{Cd} 、 \mathrm{Cr} 、 \mathrm{Cu} 、 \mathrm{~Pb}$ 和 $\mathrm{Zn}$ 含量平均值分别为 $17.2 、 2.63 、 56.9 、 32.4 、 38.4$ 和 $96.3 \mathrm{mg} / \mathrm{kg}(\mathrm{DW})$, 不同沉积物样品中的重金属元素含量变异范围较大. 6 种重金属平均含量均高于全国水 系沉积物平均值, $\mathrm{As} 、 \mathrm{Cd} 、 \mathrm{Cr}$ 和 $\mathrm{Pb}$ 含量高于湖南潮土背景值, 表现一定的污染, 尤其是洞庭湖沉积物中 $\mathrm{Cd}$ 含量超过全国水系沉积物平均值近 20 倍 (表 1).

表 1 洞庭湖表层沉积物重金属含量 $(\mathrm{mg} / \mathrm{kg}(\mathrm{DW}))$

Tab.1 Heavy metal contents in surface sediments of Lake Dongting

\begin{tabular}{|c|c|c|c|c|c|c|}
\hline 指标 & As & $\mathrm{Cd}$ & $\mathrm{Cr}$ & $\mathrm{Cu}$ & $\mathrm{Pb}$ & $\mathrm{Zn}$ \\
\hline 洞庭湖沉积物平均值 & 17.2 & 2.63 & 56.9 & 32.4 & 38.4 & 96.3 \\
\hline 洞庭湖沉积物最大值 & 49.7 & 10.0 & 129 & 80.6 & 86.0 & 203 \\
\hline 洞庭湖沉积物最小值 & 8.85 & 0.72 & 35.9 & 12.3 & 10.2 & 32.1 \\
\hline 湖南潮土背景值 [24] & 11.0 & 0.18 & 32.0 & 70.0 & 23.0 & 98.0 \\
\hline 全国水系沉积物平均值 ${ }^{[25]}$ & 9.10 & 0.14 & 38.0 & 21.0 & 25.0 & 68.0 \\
\hline
\end{tabular}

\section{2 篮分水平浓度法计算洞庭湖沉积物重金属质量基准}

2.2.1 物种笁分水平浓度的计算 $2013-2015$ 年洞庭湖共篮查出大型底栖动物 47 种 (属), 隶属于 3 门 6 纲, 其中软体动物门 19 种 (属), 环节动物门寡毛纲 7 种, 节肢动物门昆虫纲 16 种, 甲壳纲 3 种 (属), 扁形动 物门蛭纲 2 种. 根据篮分水平浓度法的要求 ${ }^{[17,26]}$, 为剔除个别物种引起的偶然误差, 选取检出率超过 $5 \%$ 的 38 种物种作为篮分物种推导沉积物重金属基准值. 出现篮分物种的沉积物样品重金属含量值进行排序, 取 
90\%的篮分物种对应的沉积物重金属含量值作为该篎分物种的篎分浓度 (species screening level concentration, SSLC), 具体见表 2 .

表 2 洞庭湖沉积物中 $\mathrm{As} 、 \mathrm{Cd} 、 \mathrm{Cr} 、 \mathrm{Cu} 、 \mathrm{~Pb}$ 和 $\mathrm{Zn}$ 的 SSLCs *

Tab.2 SSLCs for As, Cd, Cr, Cu, Pb and $\mathrm{Zn}$ in the sediments of Lake Dongting

\begin{tabular}{|c|c|c|c|c|c|c|c|}
\hline \multirow{2}{*}{ 底栖生物 } & \multirow{2}{*}{ 检出率/\% } & \multicolumn{6}{|c|}{$\mathrm{SSLC} /(\mathrm{mg} / \mathrm{kg}(\mathrm{DW}))$} \\
\hline & & As & $\mathrm{Cd}$ & $\mathrm{Cr}$ & $\mathrm{Cu}$ & $\mathrm{Pb}$ & $\mathrm{Zn}$ \\
\hline 凹狭隐摇蚊 Cryptochironomus defectus & 27.13 & 17.85 & 2.21 & 53.31 & 43.96 & 31.94 & 89.33 \\
\hline 多巴小摇蚊 Microchironomus tabarui & 16.28 & 24.01 & 2.65 & 41.96 & 20.12 & 43.98 & 80.76 \\
\hline 黄色羽摇蚊 Chironomus flaviplumus & 24.81 & 13.39 & 2.92 & 66.43 & 26.03 & 31.82 & 95.94 \\
\hline 嗀隐摇蚊 Cryptochironomus rostratus & 13.95 & 11.38 & 2.74 & 41.29 & 39.84 & 51.15 & 90.38 \\
\hline 九斑多足摇蚊 Polypedilum masudai & 8.53 & 21.85 & 1.87 & 65.93 & 38.98 & 36.83 & 58.61 \\
\hline 双线环足摇蚊 Cricotopus bicinctus & 12.40 & 38.85 & 1.92 & 72.53 & 35.73 & 30.94 & 81.21 \\
\hline 蚊型前突摇蚊 Procladius culiciformis & 20.08 & 24.73 & 2.76 & 60.54 & 30.42 & 48.57 & 93.45 \\
\hline 霞甫多足摇蚊 Polypedilum convexum & 14.73 & 29.82 & 1.11 & 45.43 & 21.63 & 42.45 & 104.40 \\
\hline 云集多足摇蚊 Polypedilum nubifer & 20.93 & 24.01 & 2.65 & 41.96 & 20.12 & 43.98 & 80.76 \\
\hline 中国长足摇蚊 Tanypus chinensis & 22.48 & 19.85 & 2.31 & 63.31 & 43.96 & 51.94 & 99.33 \\
\hline 薄壳丽蚌 Lomprotula leleaci & 5.43 & 13.25 & 3.86 & 46.94 & 18.23 & 19.26 & 73.05 \\
\hline 背角无齿蚌 Anodonta woodiana & 5.43 & 19.96 & 1.88 & 50.64 & 23.65 & 34.68 & 114.61 \\
\hline 赤豆螺 Bithynia fuchsiana & 9.30 & 10.04 & 3.72 & 72.18 & 36.27 & 17.30 & 45.44 \\
\hline 大沼螺 Parafossarulus eximius & 10.08 & 21.98 & 2.50 & 70.52 & 33.22 & 53.11 & 129.98 \\
\hline 淡水壳菜 Limnoperna lacustris & 11.63 & 31.37 & 5.01 & 48.34 & 28.16 & 55.97 & 104.69 \\
\hline 方格短沟蜷 Semisulcospira cancellata & 24.81 & 12.81 & 1.39 & 63.86 & 29.87 & 31.45 & 142.04 \\
\hline 河蚬 Corbicula fluminea & 28.68 & 10.51 & 2.79 & 53.04 & 20.63 & 27.76 & 75.18 \\
\hline 黑龙短沟蜷 Semisulcospira amurensis & 10.08 & 39.66 & 4.01 & 71.34 & 57.26 & 40.28 & 132.69 \\
\hline 湖球蚬 Sphaerium lacustre & 11.63 & 9.46 & 1.06 & 58.94 & 28.79 & 38.94 & 143.69 \\
\hline 壳菜 Limnoperna lacustris & 6.98 & 21.23 & 1.73 & 53.69 & 30.25 & 36.94 & 79.84 \\
\hline 拉氏蚬 Corbicula largillierti & 11.63 & 20.07 & 3.38 & 67.31 & 19.45 & 25.15 & 96.65 \\
\hline 梨形环棱螺 Bellamya purificata & 25.58 & 16.22 & 4.66 & 50.76 & 22.66 & 36.98 & 66.74 \\
\hline 铜锈环棱螺 Bellamya aeruginosa & 29.46 & 14.54 & 1.81 & 90.09 & 40.81 & 42.04 & 102.10 \\
\hline 圆顶珠蚌 Unio douglasiae & 20.93 & 19.71 & 3.81 & 67.21 & 32.45 & 50.49 & 86.10 \\
\hline 中国圆田螺 Cipangopaludina chinensis & 18.60 & 15.64 & 1.32 & 58.46 & 21.42 & 41.09 & 94.69 \\
\hline 淡水单孔蚓 Monopylephorus limesus & 16.28 & 17.24 & 1.04 & 43.69 & 35.69 & 43.69 & 103.69 \\
\hline 霍甫水丝蚓 Limnodrilus hoffmecisteri & 24.81 & 28.46 & 3.06 & 87.69 & 49.96 & 56.33 & 183.46 \\
\hline 巨毛水丝蚓 Limnodrilus udekemianus & 27.91 & 18.95 & 2.78 & 70.46 & 50.36 & 56.84 & 122.69 \\
\hline 前囊管水蚓 Aulodrilus prothecatus & 20.16 & 23.25 & 4.94 & 67.98 & 39.9 & 53.64 & 169.88 \\
\hline 苏氏尾鳃蚓 Branchiura soweribig & 27.91 & 24.26 & 3.13 & 89.46 & 28.64 & 54.86 & 119.98 \\
\hline 正颤蚓 Tubifex tubifex & 6.98 & 24.48 & 2.06 & 86.54 & 46.98 & 46.54 & 124.94 \\
\hline 中华颤蚓 Tubifex sinicus & 6.98 & 17.26 & 2.31 & 69.93 & 51.36 & 43.69 & 119.69 \\
\hline 蜉游 Ephemera sp. & 13.18 & 28.96 & 0.96 & 38.96 & 19.84 & 27.43 & 63.25 \\
\hline 低头石虫 Neureclipsis sp. & 20.16 & 17.92 & 2.03 & 54.69 & 26.54 & 35.64 & 71.96 \\
\hline 钩虾 Gammarus sp. & 20.93 & 23.36 & 1.08 & 65.69 & 23.39 & 28.94 & 71.24 \\
\hline 箭蜓 Gomphus sp. & 8.53 & 15.69 & 2.32 & 51.79 & 33.64 & 31.97 & 76.94 \\
\hline 蠓蚊 Ceratopogonidae sp. & 20.16 & 11.24 & 1.32 & 50.31 & 31.62 & 31.29 & 69.81 \\
\hline 蛭类 Hirudinea sp. & 23.26 & 19.36 & 1.87 & 66.32 & 32.45 & 33.64 & 96.46 \\
\hline
\end{tabular}

* 检出率为样品中物种的出现次数与总有效样本数的百分比 (包括不同采样站点, 或同一采样站点不同采样时间获取的 样品).

2.2.2 篮分水平浓度的计算 不同物种的 SSLC 按照从小到大的顺序进行排序, 并进行正态分布检验, 结果 
表明 $\mathrm{As} 、 \mathrm{Cd} 、 \mathrm{Cr} 、 \mathrm{Cu} 、 \mathrm{~Pb}$ 和 Zn 6 种重金属元素的物种篎分水平浓度 SSLC 均符合正态分布( 表 3 ).

表 3 洞庭湖沉积物中 $\mathrm{As} 、 \mathrm{Cd} 、 \mathrm{Cr} 、 \mathrm{Cu} 、 \mathrm{~Pb}$ 和 $\mathrm{Zn}$ 的 SSLC 分布检验

Tab.3 Normality test of SSLCs for As, Cd, Cr, Cu, Pb and $\mathrm{Zn}$ in the sediments of Lake Dongting

\begin{tabular}{lcccccc}
\hline 统计量 & As & $\mathrm{Cd}$ & $\mathrm{Cr}$ & $\mathrm{Cu}$ & $\mathrm{Pb}$ & $\mathrm{Zn}$ \\
\hline 样本量 & 38 & 38 & 38 & 38 & 38 & 38 \\
Shapiro-Wilk 检验(检验水平为 0.05) & 0.06 & 0.06 & 0.08 & 0.06 & 0.35 & 0.09 \\
Kolmogorov-Smirnov 检验(检验水平为 0.05) & 0.69 & 0.80 & 0.89 & 0.97 & 0.98 & 0.48 \\
\hline
\end{tabular}

利用表 2 中的数据, 做出洞庭湖沉积物 $\mathrm{As} 、 \mathrm{Cd} 、 \mathrm{Cr} 、 \mathrm{Cu} 、 \mathrm{~Pb}$ 和 $\mathrm{Zn}$ 的物种耖分浓度的百分位图, 并利用 $\mathrm{Or}-$ igin 8.5 软件对其进行正态拟合,根据不同物种的 SSLC 拟合结果,选择 5\% (即 95\%的物种得到保护) 对应的 $\mathrm{As} 、 \mathrm{Cd} 、 \mathrm{Cr} 、 \mathrm{Cu} 、 \mathrm{~Pb}$ 和 $\mathrm{Zn}$ 含量作为洞庭湖沉积物中各种金属笁分水平浓度 (screening level concentration, SLC) (图 2), 最终获取洞庭湖沉积物 $\mathrm{As} 、 \mathrm{Cd} 、 \mathrm{Cr} 、 \mathrm{Cu} 、 \mathrm{~Pb}$ 和 $\mathrm{Zn}$ 的 SLC 分别为 11.97、1.13、42.03、19.17、23.63 和 $67.54 \mathrm{mg} / \mathrm{kg}(\mathrm{DW})$, 即可作为洞庭湖沉积物 6 种重金属的基准值.
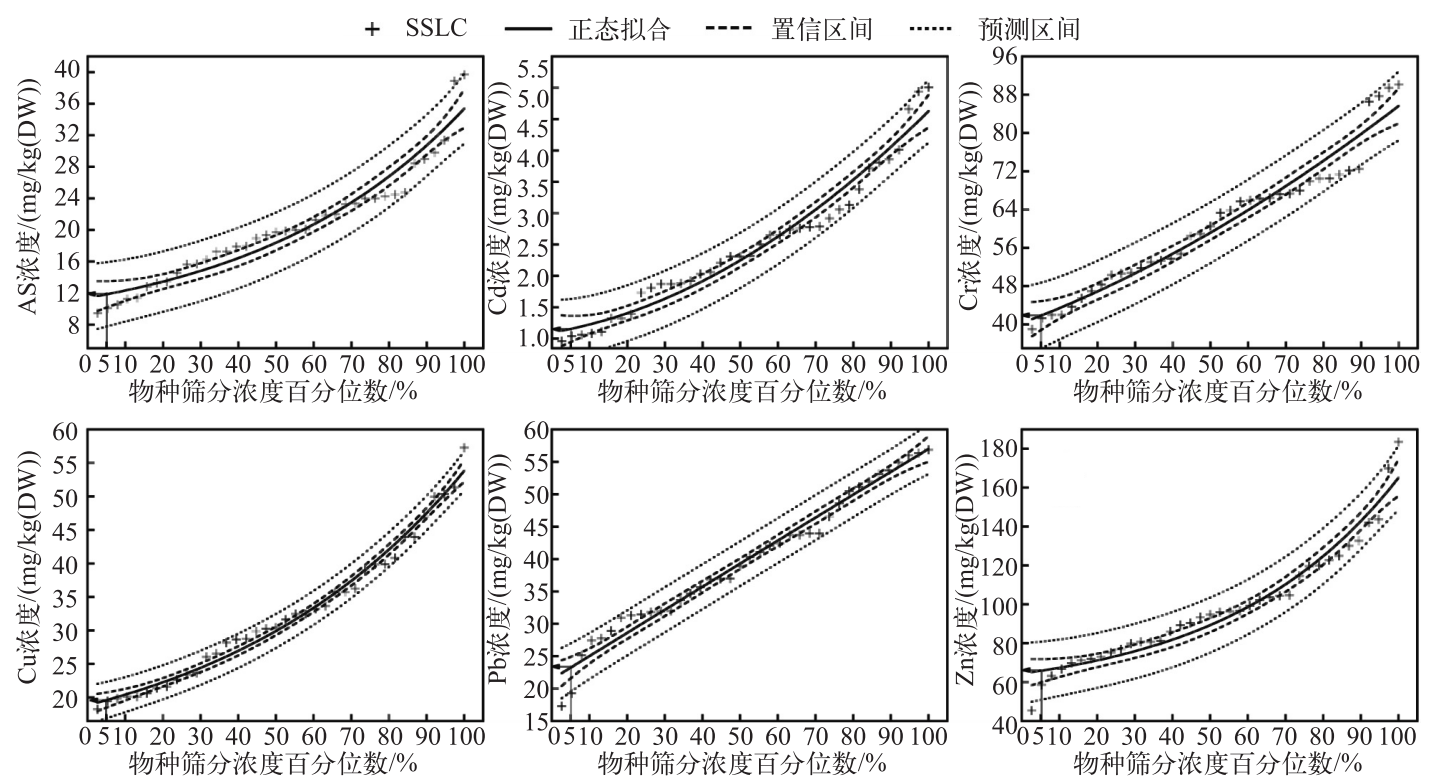

图 2 推导洞庭湖沉积物 $\mathrm{As} 、 \mathrm{Cd} 、 \mathrm{Cr} 、 \mathrm{Cu} 、 \mathrm{~Pb}$ 和 $\mathrm{Zn}$ 的 SLCs

Fig.2 SLCs of $\mathrm{As}, \mathrm{Cd}, \mathrm{Cr}, \mathrm{Cu}, \mathrm{Pb}$ and $\mathrm{Zn}$ for the sediments of Lake Dongting

\section{3 讨论}

\section{1 不同国家和地区的沉积物重金属基准值}

不同国家和地区的沉积物重金属基准存在明显差异, 除 Zn 基准最大值和最小值的差异小于 10 倍之 外,其他重金属基准值差异均超过 10 倍,其中 $\mathrm{Cd}$ 基准最大值和最小值之比达到 60 倍, $\mathrm{Cu}$ 基准最大值和最 小值之比也超过 40 倍(表 4). 基准差异产生的主要原因在于各国家和地区制定沉积物质量基准的方法各不 相同, 保护目标和保护程度也有差异, 因此在笁选环境因子以及获得生物效应数据方面会产生差异 ${ }^{[8]}$. 本研 究基于笁分水平浓度法获取的洞庭湖沉积物 $\mathrm{Cd} 、 \mathrm{Cu} 、 \mathrm{~Pb} 、 \mathrm{Zn} 4$ 种重金属的基准值与霍文毅等 ${ }^{[27]}$ 基于相平衡 分配法获取的洞庭湖沉积物基准值大体相当, 表明同一区域内不同方法获取的沉积物重金属基准值具有可 比性.

然而, 由于沉积物中重金属的生物效应受到诸多因素的制约和影响, 这些影响因素又随沉积物类型和 
环境条件的改变而表现出较大的不同,导致本研究获取的沉积物质量基准仍然存在较大的不确定性. 首先, 由于重金属的生物毒性主要取决于形态而非总量 ${ }^{[28]}$, 而特定重金属在水环境中的迁移转化过程中通常伴随 着形态改变,篮分水平浓度法获取沉积物质量基准无法区分不同形态的重金属对生物效应的贡献. 此外, 不

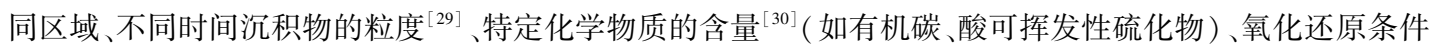
往往存在差异,造成了应用沉积物质量基准进行沉积物质量质量评价的不确定性.

表 4 不同国家和地区沉积物重金属质量基准值 $(\mathrm{mg} / \mathrm{kg}(\mathrm{DW}))^{*}$

Tab.4 Sediment quality criteria values for heavy metals in different countries or areas

\begin{tabular}{lccccccc}
\hline 沉积物质量基准 & 来源 & $\mathrm{As}$ & $\mathrm{Cd}$ & $\mathrm{Cr}$ & $\mathrm{Cu}$ & $\mathrm{Pb}$ & $\mathrm{Zn}$ \\
\hline 洞庭湖基准 & 本研究 & 11.97 & 1.13 & 42.03 & 19.17 & 23.63 & 67.54 \\
洞庭湖基准 & {$[31]$} & - & 0.16 & - & 19.00 & 10.00 & 56.00 \\
鄱阳湖基准 & {$[27]$} & - & 0.33 & - & 46.00 & 18.00 & 64.00 \\
黄河中游基准 & {$[27]$} & - & 1.30 & - & 23.00 & 17.00 & 98.00 \\
长江中下游基准 & {$[27]$} & - & 1.70 & - & 650.00 & 250.00 & 300.00 \\
太湖基准 & {$[32]$} & - & 6.42 & - & 55.30 & 20.60 & 201.50 \\
辽河基准 & {$[32]$} & - & 5.42 & - & 52.80 & 18.90 & 177.70 \\
NOAA 的 ERL & {$[33]$} & 8.20 & 1.20 & 81.00 & 34.00 & 46.70 & 150.00 \\
NOAA 的 ERM & {$[33]$} & 70.00 & 9.60 & 370.00 & 270.00 & 218.00 & 410.00 \\
佛罗里达州 LEL & {$[34]$} & 6.00 & 0.60 & 26.00 & 16.00 & 31.00 & 120.00 \\
佛罗里达州 PEL & {$[34]$} & 41.60 & 4.21 & 160 & 108 & 112 & 271 \\
USEPA 的 TEL-HA28 & {$[35]$} & 11.00 & 0.58 & 36.00 & 28.00 & 37.00 & 98.00 \\
USEPA 的 PEL-HA28 & {$[35]$} & 48.00 & 3.20 & 120.00 & 100.00 & 82.00 & 540.00 \\
最大值 & & 70.00 & 9.60 & 370.00 & 650.00 & 250.00 & 540.00 \\
最小值 & & 6.00 & 0.16 & 26.00 & 16.00 & 18.90 & 56.00 \\
最大值/最小值 & & 11.67 & 60.00 & 14.23 & 40.63 & 13.23 & 9.64 \\
\hline
\end{tabular}

$* \mathrm{ERL}$ 为效应范围低值; ERM 为效应范围中值; LEL 为最低效应水平; PEL 为可能效应水平; TEL-HA28 和 PEL-HA28 为 $28 \mathrm{~d}$ Hyalella azteca 阈值效应水平阈值和可能效应水平阈值; NOAA 为美国大气与海洋管理局; FDEP 为美国佛罗里达州 环境保护局.

\section{2 篮分水平浓度法推导沉积物质量基准的分析与建议}

与背景值法 ${ }^{[1]}$ 、相平衡分配法 ${ }^{[12,14]}$ 和沉积物加标毒性实验法 ${ }^{[8,36]}$ 等沉积物质量推导方法相比,篮分水 平浓度法的优势在于: (1) 适用范围广. 笁分水平浓度法通过获取相互匹配的沉积物和底栖生物调查数据, 经过统计分析获取沉积物质量基准, 对污染物的类型以及沉积物的种类无特殊限定, 可用于各种类型污染 物 (重金属、持久性有机污染物) 的沉积物质量基准推导; (2) 在一定程度上反映了污染物的生物毒性. 不同 污染物之间相加、拮抗、协同关系最终以对底栖生物综合毒性的方式作用于底栖生物, 而篮分水平浓度法以 底栖生物物种出现与否作为对沉积物综合毒性的反应; (3) 方法简单, 具有很强的可操作性. 笁分水平浓度 法能够在不需要额外增加相关分析的基础上, 直接利用监测数据推导沉积物质量基准. 作为构建沉积物质 量基准的主流方法之一, 加拿大安大略省利用篮分水平浓度法构建了包括金属、PCBs 和有机氯杀虫剂的沉 积物质量基准 ${ }^{[37-38]}$. 篮分水平浓度法的缺点则包括: (1) 难以辨别其他因素的影响, 如人工或天然因素造成 的底栖生物栖息地环境改变; (2) 难以明确污染物和生物效应之间的因果关系; (3) 以底栖生物出现与否作 为评判指标过于 “激进”, 缺乏对亚致死剂量的考虑; (4) 篮分水平浓度法推导的沉积物质量基准的合理与 否, 在很大程度上取决于监测数据的丰富程度, 对于特定流域, 数据不足将会导致较大的不确定性. USEPA 以篮分水平浓度法推导沉积物质量基准时, 要求每种物种在观测站位中出现频率超过 10 次, 而用于推导 SLC 的 SSLC 数不少于 20 个 $^{[39]}$.

本研究中受限于调查范围以及调查方法. 本研究获取的数据有限, 而且洞庭湖人类开发活动尤其是洞 庭湖大量采砂, 严重干扰了底栖生物的正常分布, 导致调查数据有所偏差, 因此本研究获取的洞庭湖沉积物 重金属的质量基准具有一定的不确定性. 随着我国监测体系的完善, 尤其是 “十二五” 期间, 我国生物监测网 
络日趋完善 ${ }^{[40]}$, 用于推导沉积物质量基准的相互匹配的化学数据和生物调查数据逐渐丰富, 沉积物质量基 准推荐值的不确定性有望降低. 此外, 针对笁分水平浓度以底栖动物出现与否作为评判指标过于 “激进” 的 问题, 可适当选择篮分物种进行沉积物加标毒性试验, 补充个体、组织、细胞乃至分子水平的效应的相关结 果,降低基准的不确定性.

目前我国沉积物质量基准研究尚处于起步阶段,笁分水平浓度法作为一种计算简单、适用性强、能够充 分利用监测数据且获取的结果能够反映底栖生物对污染物反应的沉积物质量基准推导方法, 在水环境管理 中具有较强的应用价值. 然而笁分水平浓度法也存在一定的局限和不足, 未来工作中应着力在以下几个方 面进行补充和完善, 提高沉积物质量基准: (1) 建立沉积物生物效应综合数据库, 加强各学科各部门数据的 互联互通. 笁分水平浓度法的精准度取决于数据的数量和质量, 针对我国水环境监测数据掌握在环保、水 利、农业等不同部门的现状, 有必要在相关数据进行检验、校正和可靠性评估的基础上, 进行数据整合, 建立 统一的沉积物生物效应综合数据库, 为推导沉积物质量基准奠定基础. (2) 重视沉积物化学分析、毒性实验 和生物调查的结合研究, 建立沉积物污染和生物效应之间的因果关系, 提高基准的可靠性. (3) 加强对沉积 物中污染物生物毒性的影响因素研究, 如开展沉积物粒度、有机碳、酸可挥发性硫化物等因素与沉积物中污 染物毒性的相关关系研究,为基准的标准化校准提供依据.

\section{4 结论}

洞庭湖沉积物重金属元素含量高于全国水系沉积物平均值, 表现出一定的污染, 其中镉污染程度最高. 应用篮分水平浓度法获取了洞庭湖沉积物 $\mathrm{As} 、 \mathrm{Cd} 、 \mathrm{Cr} 、 \mathrm{Cu} 、 \mathrm{~Pb}$ 和 $\mathrm{Zn}$ 的基准值, 分别为 11.97、1.13、42.03、 19.17、23.63 和 $67.54 \mathrm{mg} / \mathrm{kg}$. 同一区域应用不同沉积物质量基准推导方法获取的重金属基准值具有可比性. 然而, 受限于调查数据以及沉积物质量基准推导方法自身的局限性, 获取的沉积物质量基准值具有一定的 不确定性.

致谢: 南开大学生命科学学院王新华教授在物种鉴定方面提供大量帮助, 在此表示感谢.

\section{5 参考文献}

[ 1 ] Förstner U, Wittmann GTW eds. Metal pollution in the aquatic environment. Berlin: Springer-Verlag, 1979:110-192.

[ 2 ] Rognerud S, Fjeld E. Trace element contamination of Norwegian Lake sediments. Ambio, 2001, 30(1):11-19.

[ 3 ] Yan Ting, Liu Enfeng, Zhang Enlou et al. The spatio-temporal variations of heavy metals in the sediment of Lake Fuxian and the contamination assessment. J Lake Sci, 2016, 28(1):50-58. DOI:10.18307/2016.0106. [燕婷, 刘恩峰, 张恩 楼等. 抚仙湖沉积物重金属时空变化与人为污染评价. 湖泊科学, 2016, 28(1): 50-58.]

[ 4 ] Chen Jingsheng, Wang Feiyue. Some problems on sediment quality criteria. Environmental Chemistry, 1992, 11(3):6070. [ 陈静生, 王飞越. 关于水体沉积物质量基准问题. 环境化学, 1992, 11(3)：60-70.]

[ 5 ] DiToro DM, McGrath JA. Technical basis for narcotic chemicals and polycyclic aromatic hydrocarbon criteria II . Mixtures and sediments. Environmental Toxicology and Chemistry, 2000, 19(8): 1951-1970.

[ 6 ] Chapman PM, Alland PJ, Vigers GA. Development of sediment quality values for Hong Kong Special Administrative Region: A possible model for other jurisdictions. Marine Pollution Bulletin, 1999, 38(3) : 161-169.

[ 7 ] US EPA. A Guidance manual to support the assessment of contaminated sediments in freshwater ecosystem great lakes national program office. Chicago: Illinois, 2002, EPA-905-B02-001-B.

[ 8 ] Chen Yunzeng, Yang Hao, Zhang Zhenke et al. The difference and cause analyses of freshwater sediment quality guidelines. J Lake Sci, 2005, 17(3) :193-201. DOI: 10.18307/2005.0301. [陈云增, 杨浩, 张振克等. 淡水沉积物环境质 量基准差异分析. 湖泊科学, 2005, 17(3): 193-201.]

[ 9 ] Liu Wenxin, Luan Zhaokun, Tang Hongxiao. Sediment quality criteria for heavy metal pollution in the Lean River II. Equilibrium partitioning approach. Acta Scientiae Circumstantiae, 1999, 19(3): 230-235. [刘文新, 奕兆坤, 汤鸿霄. 河流 沉积物重金属污染质量控制基准的研究 II. 相平衡分配方法 (EqP). 环境科学学报, 1999, 19(3)：230-235.]

[10] Wen Xianghua. Sediment quality criteria for heavy metals. Environmental Chemistry, 1993, 12(5): 334-341. [ 文湘华. 水体沉积物重金属质量基准研究. 环境化学, 1993, 12(5): 334-341.]

[11] Wang Lixin, Chen Jingsheng. The advance of approaches for deriving sediment quality criteria for heavy metals. Acta Scien- 
tianrum Naturalium Universitatis NeiMongl, 2003, 34(4): 472-477. [王立新, 陈静生. 建立水体沉积物重金属质量 基准的方法研究进展. 内蒙古大学学报: 自然科学版, 2003, 34(4): 472-477.]

[12] Fang Tao, Xu Xiaoqing. Establishment of sediment quality criteria for metals in water of the Yangte River using equilibrium-partitioning approach. Resources and Environment in the Yangtze Basin, 2007, 16(4) : 525-531. [方涛, 徐小清. 应 用平衡分配法建立长江水系沉积物金属相对质量基准. 长江流域资源与环境, 2007, 16(4): 525-531.]

[13] Zhang Ting, Zhong Wenyu, Zeng Yi et al. Sediment heavy mentals quality criteria for fresh waters based on biological effect database approach. Chinese Journal of Applied Ecology, 2012, 23(9): 2587-2594. [张婷, 钟文理, 曾毅等. 应用 生物效应数据库法建立淡水水体沉积物重金属质量基准. 应用生态学报, 2012, 23(9): 2587-2594.]

[14] Zhu Lingyan, Liu Nannan, Deng Baole. Sediment quality criteria for organic pollutants based on phase-equilibrium partitioning approach: A review. Chinese Journal of Applied Ecology, 2009, 20(10): 2574-2580. [祝凌燕, 刘楠楠, 邓保 乐. 基于相平衡分配法的水体沉积物中有机物污染物质量基准研究进展. 应用生态学报, 2009, 20(10): 2574-2580. ]

[15] Zhong Wenyu, Chang Chun, Zeng Yi et al. Development of freshwater sediment quality criteria for nonionic organics-Using Lindan as an example. Asian Journal of Ecotoxicology, 2011, 6(5): 476-484. [ 钟文理, 常春, 曾毅等. 非离子有 机物淡水沉积物质量基准推导方法——以林丹为例. 生态毒理学报, 2011, 6(5): 476-484.]

[16] Chen Yunzeng, Yang Hao, Zhang Zhenke et al. Reiview of approaches for deriving sediment quality guidelines. Advances in Earth Science, 2006, 21 (1) : 53-61. [ 陈云增, 杨浩, 张振克等. 水体沉积物环境质量基准建立方法研究进展. 地 球科学进展, 2006, 21(1): 53-61.]

[17] Neff JM, Bean DJ, Comaby BW et al. Sediment quality criteria methodology validation calculation of screening level concentration from field data. Washington DC: Office of Water Regulation and Standards, 1986.

[18] Zhao Yanmin, Zhang Lei, Qin Yanwen et al. Sediment quality critera for heavy metals in Hunhe River based on screening level concentration approach. Asian Journal of Ecotoxicology, 2014, 9(2) : 329-338. [赵艳民, 张雷, 秦延文等. 筛分 水平浓度法构建浑河沉积物重金属基准初步研究. 生态毒理学报, 2014, 9(2): 329-338. ]

[19] Zhu Yunlong, Jiang Jiahu, Sun Zhandong et al. Character and assessment of heavy metals in the sediments from Lake Dongting. J Lake Sci, 20(4) : 477-485. DOI: 10.18307/2008.0411. [祝云龙, 姜加虎, 孙占东等. 洞庭湖沉积物中重 金属污染特征与评价. 湖泊科学, 2008, 20(4): 477-485.]

[20] Wang Qiuheng, Wang Shuyun, Liu Meiying. Saftey evaluation on pollution of Xiang River valley in Hunan Province. China Water \& Waste Water, 2004, 20(8) : 104-106. [王秋衡, 王淑云, 刘美英. 湖南湘江流域污染的安全评价. 中国给水 排水, 2004, 20(8): 104-106.]

[21] Zhang Guanggui, Huang Bo. Health risk assessment of heavy metals in Dongting Lake water system in Hunan Province, China. Water Resources Protection, 2014, 30(1): 14-17, 47. [张光贵, 黄博. 湖南洞庭湖水系沉积物重金属健康风 险评价. 水资源保护, $2014, \mathbf{3 0}(1): 14-17,47$.

[22] Dong Meng, Zhao Yunlin, Lei Cunxi et al. Distribution and pollution evaluation of four kinds of heavy metals in chau embankment soil of South Dongting Lake. Soils, 2010, 42(3) : 453-458. [董萌, 赵运林, 雷存喜等. 南洞庭湖洲垸土壤 中四种重金属的分布特征及污染状况评价. 土壤，2010，42(3) : 453-458.]

[23] Wan Qun, Li Fei, Zhu Huina et al. Distribution characteristics, pollution assessment and source identification of heavy metals in the sediment of east Dongting Lake. Research of Environmental Sciences, 2011, 24(12): 1378-1384. [万群, 李 飞, 祝慧娜等. 东洞庭湖沉积物中重金属的分布特征、污染评价与来源辨析. 环境科学研究, 2011, 24(12)： 1378-1384. ]

[24] Li Yongjin, Tang Yuxi, Tang Jie et al. Distribution and ecological risk assessment of heavy metal elements in soils of the beaches in Dongting Lake. Journal of Central South University of Forestry \& Technology, 2011, 31(2) : 55-59. [ 李永进, 汤玉喜, 唐洁等. 洞庭湖滩地重金属分布及其生态风险评价. 中南林业科技大学学报: 自然科学版, 2011, 31 (2) : 55-59.]

[25] Yan Mingcai, Chi Qinghua, Gu Tiexin et al. Average element content of various sediments in China. Geophysical \& Geochemical Exploraion, 19(6): 468-472. [ 鄢明才, 迟清华, 顾铁新等. 中国各类沉积物化学元素平均含量. 物探与化 探, $1995,19(6): 468-472$.

[26] US EPA. Sediment quality criteria methodology validation: Calculation of screening level concentrations from field data. Washington DC: Office of Water, 1986. 
[27] Huo Wenyi, Chen Jingsheng. Water-particulate distribution coefficient of heavy metal and application in sediment quality criteria in China River. Environmental Sciences, 1997, 18(4):10-13. [霍文毅, 陈静生. 我国部分河流重金属水-固分 配系数及在河流质量基准研究中的应用. 环境科学, 1997, 18(4) : 10-13.]

[28] Lee CF, Jones-Lee A. Can chemically-based sediment quality criteria be used as reliable screening tools for water quality impacts? Society of Environmental Toxicology and Chemistry News, 1996, 16(3) : 14-15.

[29] Horowitz AJ, Elrick KA. The relation of stream sediment surface area, grain size, and compostion to trace element chemistry. Applied Geochemistry, 1987, 2(4) : 437-451.

[30] Di Toro DM, Mahony JD, Hansen DJ et al. Toxicity of cadmium in sediments: The role of acid volatile sulfide. Environmental Toxicology and Chemistry, 1990, 9(12): 1487-1502.

[31] Wang Feiyue. Environmental Geochemistry of particulate matter and heavy metals in eastern China[Dissertation]. Beijing: Peking University, 1995. [王飞越. 中国东部河流颗粒物-重金属环境地球化学 [学位论文]. 北京: 北京大 学, 1995.]

[32] Deng Baole, Zhu Lingyan, Liu Man et al. Sediment quality criteria and ecological risk assessment for heavy metals in Taihu Lake and Liao River. Research of Environmental Sciences, 2011, 24(1) : 33-42. [邓保乐, 祝凌燕, 刘慢等. 太湖和辽 河沉积物重金属质量基准及生态风险评估. 环境科学研究, 2011, 24(1):33-42.]

[33] NOAA. Screening Quick Reference Tables (SQuiRT). Washington DC: Coastal Protection and Restoration Division, 1999.

[34] MacDonald DD. Approach to the assessment of sediment quality in Florida coastal waters. Tallahassee, Florida: Florida Department of Environmental Protection Office of Water Policy, 1994.

[35] MacDonald DD, Ingersoll CG, Berger TA. Development and evaluation of consensus-based sediment quality guidelines for freshwater ecosystems. Archives of Environmental Contamination and Toxicology, 2000, 39(1) : 20-31.

[36] Swartz RC, Kemp PF, Schults DW et al. Acute toxicity of sediment from Eagle Harbour, Washington, to the infaunal amphipod Rhepoxynius abronius. Environmental Toxicology and Chemistry, 1995, 8(3): 215-222.

[37] Jaagumagi R. Development of the ontario provincial sediment quality guidelines for Arsenic, Cadmium, Chromium, Copper, Iron, Lead, Manganese, Mercury, Nickel, and Zinc. Toronto: Water Resources Branch, Environment Ontario, 1990.

[38] Jaagumagi R. Development of the ontario provincial sediment quality guidelines for PCBs and the organochlorine pesticides. Toronto: Water Resources Branch, Environment Ontario, 1990.

[39] Chapman PM. Current approaches to developing sediment quality criteria. Environmental Toxicology and Chemistry, 1989, 8( 7$)$ : 589-599.

[40] Yin Kun, Lü Yibing, Teng Enjiang. American water environment bio-monitoring system and its suggestion for bio-monitoring in China. The Administration and Technique of Environmental Monitoring, 2012, 24(5): 8-12. [阴琨, 吕怡兵, 滕 恩江. 美国水环境生物监测体系及对我国生物监测的建议. 环境监测管理与技术, 2012, 24(5): 8-12.] 